\title{
Critério diagnóstico provisório para depressão na doença de Alzheimer
}

Olin J.T., Schneider L.S., Kats I.R., Meyers B.S. Alexopoulos G.S. et al. Provisional Diagnostic criteria for depression in Alzheimer's disease. American Journal of Geriatric Psychiatry 10: 125-8, 2002

Comentado por Mario Cesar de Souza Bazzarella ${ }^{1}$

Recebido: 26/6/2003 Aceito: 27/6/2003

Um grupo de pesquisadores publicou recentemente um artigo descrevendo um critério diagnóstico provisório para depressão na doença de Alzheimer (DA) (Olin et al., 2002). Um avanço nesse campo de estudo foi a caracterização dos sintomas de comportamento apresentados pelos pacientes com demência através do termo BPSD (Behavioral and psychological symptoms of dementia ou sintomas psicológicos e comportamentais da demência). No entanto, este conceito foi considerado muito amplo para se definir uma estratégia terapêutica, resultando em uma tendência para descrever síndromes específicas em pacientes com DA. A psicose da DA foi melhor definida recentemente, sendo considerada um alvo potencial para intervenção (Jeste e Finkel, 2000). A depressão da DA é outra síndrome, presente no conceito BPSD, que necessita ser melhor caracterizada, dada a alta freqüência desses sintomas em pacientes com DA, podendo causar aumento no custo do tratamento, maior sofrimento dos familiares e cuidadores e institucionalização precoce.

Embora o Manual estatístico e diagnóstico de doenças mentais (DSM-IV-TR) (APA, 2000) possa ser usado para identificar a depressão como um diagnóstico isolado do eixo I, coexistindo com a DA, os critérios para permitir essa separação não foram definidos. Por exemplo, uma característica como "perda de interesse" não está especificada e pode ser observada em pacientes com depressão clinicamente significativa ou em pacientes com demência e distúrbio de humor não aparente. Também existem dificuldades para o uso dos critérios descritivos, tais como aqueles encontrados no transtorno depressivo maior ou no transtorno depressivo menor, para caracterizar a depressão encontrada em pacientes com DA.

O DSM-IV-TR (APA, 2000), assim como os manuais de classificação anteriores, foi desenvolvido para pacientes com condições psiquiátricas idiopáticas, e sua utilização depende muito da habilidade verbal dos pacientes para relatar os sintomas depressivos subjetivos, tais como mudança de humor, perda de interesse, ou desesperança. A expressão verbal é habitualmente menor nos pacientes com DA, e o retraimento e o isolamento social observados em indivíduos deprimidos com DA devem ser diferenciados dos comportamentos que ocorrem no contexto da demência.

De acordo com Olin et al. (2002), embora os critérios de diagnóstico provisório sejam derivados dos critérios para o episódio depressivo maior, existem várias diferenças significativas. Primeiro, a depressão da DA requer a presença de três ou mais sintomas (contra cinco ou mais para episódio depressivo maior). Segundo, não há a necessidade da ocorrência dos sintomas quase que diariamente, como no caso do episódio depressivo maior. Terceiro, foram acrescentados critérios para a presença de irritabilidade e de isolamento e retraimento social. Em quarto lugar, os critérios para perda de interesse ou prazer foram revisados para mostrar diminuição de atitude positiva ou prazer, em resposta a contato social ou atividades cotidianas.

Diferença potencialmente relevante entre a depressão da DA e a depressão primária pode envolver incidência e prevalência, história prévia, história familiar, gravidade, freqüência do episódio de depressão maior, suicídio, duração dos episódios, e resposta terapêutica. Por exemplo, a depressão da DA pode ser menos grave

\footnotetext{
Médico Psiquiatra. Colaborador do PROTER - Instituto de Psiquiatria do HCFMUSP

Endereço para correspondência:

Instituto e Departamento de Psiquiatria da FMUSP

Rua Dr. Ovídio Pires de Campos s/n, São Paulo, SP - CEP 05403-010

E-mail: cbottino@usp.br
}

Olin, J.T.; Schneider, L.S.; Kats, I.R.; Meyers, B.S.; Alexopoulos, G.S. et al.

Rev. Psiq. Clín. 30 (3):102-103, 2003 
ou persistente, com flutuação da sintomatologia. Pode haver menos ideação suicida e menos tentativas de suicídio, assim como menos sintomas melancólicos. Deve-se considerar, também, que sintomas de depressão, tais como: perda de peso, alterações do sono ou fadiga em pacientes com demência podem resultar da coexistência com outras doenças médicas.

O diagnóstico da depressão da DA é feito, primariamente, por meio da avaliação clínica cuidadosa, que é mais importante do que a aplicação de escalas de classificação de sintomas. O clínico deve estabelecer o diagnóstico de demência do tipo Alzheimer e depois identificar se existem sintomas depressivos clinicamente significativos. Isto envolve uma avaliação clínica abrangente e detalhada, considerando as associações temporais entre o início e o curso da depressão e da demência. Finalmente, deve-se julgar se a depressão não é melhor explicada por uma depressão primária, outras doenças mentais, outras condições médicas, ou efeitos adversos de medicação.
Portanto, considerável julgamento clínico é necessário para fazer o diagnóstico de indivíduos com DA, cuja depressão pode ser diferenciada do transtorno depressivo maior e do transtorno depressivo menor. Os critérios provisórios descritos por Olin et al. (2002) servem como ponto de partida para tentar definir critérios operacionais mínimos que possam ser utilizados em pesquisas futuras e sirvam para avaliar a eficácia de tratamentos dos pacientes portadores de DA e depressão.

\section{Referências bibliográficas}

American Psychiatric Association. - Diagnostic and Statistical Manual of Mental Disorders, 4th. ed., Text Revision. American Psychiatric Press, Washington, DC, 2000.

Jeste, D.V.; Finkel, S.I. - Psychosis of Alzheimer's Disease and Related Dementias. Diagnostic Criteria for a Distinct Syndrome. American Journal of Geriatric Psychiatry 8: 29-34, 2000.

Olin, J.T.; Schneider, L.S.; Kats, I.R.; Meyers, B.S.; Alexopoulos, G.S.; et al. - Provisional Diagnostic Criteria for Depression in Alzheimer's Disease. American Journal of Geriatric Psychiatry 10: 125-8, 2002. 